Atmósfera 30(2), i-ii (2017)

doi: 10.20937/ATM.2017.30.02.00

\title{
Preface to the thematic issue on Climate, economics and statistics
}

Environmental problems are typically produced by the interactions of complex natural and human systems and can entail far-reaching and sometimes irreversible consequences. Their study is characterized by the existence of incomplete knowledge and high levels of uncertainty and, in some cases, ambiguousness in the definition of the problem itself and its boundaries. These socalled wicked problems openly challenge the traditional disciplinary approach commonly used in natural and social sciences and call for the development of interdisciplinary, integrated research, as well as for innovative methods and frameworks.

Recognizing the importance of fostering modern interdisciplinary research, the Editors of Atmósfera decided to broaden the scope of the journal to reach a larger research community and readership interested in different aspects of atmospheric and environmental issues. This thematic issue represents the first step in this direction and offers the reader a collection of contributions from leading authors that integrates climate, economics and statistics. The research articles can be grouped in three broad topics: the analysis of observed warming trends in temperature series and the study of its drivers; the analysis and modeling of the impacts of current and future climate and the identification of possible adaptation strategies; and the transition to a low-carbon economy.

The area average of climate variables is commonly used to describe observed trends in a changing climate. However, this representation of the warming trend may not be particularly informative for many applications, such as investigating the relationship between economic activity and climate. Based on methods originally developed to measure price inflation, Tol proposes a novel approach for computing population-weighted climate trends that considers changes in population over time. The author illustrates the use of this method by computing different estimates of the mean annual global temperature that the average person experienced during the observed period. This population-weighted warming trend is substantially modified by the effects of urbanization and to a lesser degree by international migration.

Estrada, Martins and Perron apply a recently developed attribution method to investigate the features of the underlying warming trend in sea and land, global and hemispheric temperature series. In their contribution, the authors argue that natural variability can severely distort the warming trend and that to investigate its features and drivers, the underlying trend should be purged from the observed temperature series. Two controversial issues regarding the attribution of climate change are addressed: the existence and causes of the recent slowdown in the warming and the attribution of the observed trend and its rapid changes shown by the Inter-hemispheric Temperature Asymmetry.

Climate is a determinant factor for many human and natural systems. The study of the impacts of present and future climate can be a critical input for policy-making and for developing adaptation strategies. Mendelsohn and Wang, investigate the role of climate and soils on the success of the Green Revolution in China. Their results suggest that natural endowments can be even more relevant than knowledge and market access for determining the success of high input agriculture. A likely adaptation strategy farmers may adopt under climate change would be to modify input intensity depending on changes in farm productivity.

Economic losses from natural disasters are the product of complex interactions between extreme weather and the exposure and vulnerability of human systems. As such, it is expected that the risk currently faced by different societal actors will significantly increase during this century 
due to economic and population growth as well as to changes in climate. Haer, Botzen, ZavalaHidalgo, Cusell and Ward analyze the current and future flood risks for Tabasco, Mexico, and the economic desirability of different adaptation strategies. This contribution, based on a state-of-theart multidisciplinary modeling approach, provides policy-relevant information about the costs of flooding and what the economically optimal flooding protection could be.

Investigating the relationship between economic growth and greenhouse gases emissions over the observed period and how a long-term low-carbon society could be reached entails significant modeling challenges. Jardón, Kuik and Tol analyze if economic growth in Latin America and the Caribbean led to a decrease in $\mathrm{CO}_{2}$ emissions, as economic theory suggests. This work is the first to study the existence of an environmental Kuznets curve in the region using some of the latest advances in panel unit root and co-integration tests. Schinko, Bachner, Schleicher and Steininger offer an in-depth review of the state-of-the-art energy- and climate-economic models, which focuses on their ability to successfully develop and assess pathways towards a low-carbon society. Based on their findings, the authors propose a multi-purpose integrated modeling framework that could help guiding decision-making on how to achieve the long-term emissions reductions consistent with current climate policy goals.

The contributions selected for this thematic issue combine concepts, frameworks, models and methods from different disciplines to provide novel ways to approach different aspects of the causes and consequences of climate change. We believe that Climate, economics and statistics offers the readership of Atmósfera an interesting and enriching view of climate change topics that are traditional and, in some cases, new to the journal.

\section{Francisco Estrada}

Guest Editor

Carlos Gay-García

Editor in Chief

René Garduño-López

Associated Editor 